\title{
The expression of heat shock protein A12B (HSPA12B) in non-Hodgkin's lymphomas
}

\author{
Yuejiao Huang ${ }^{1,2 \#}$, Chunlei Peng ${ }^{1 \#}$, Jie Tang $^{2}$, Shitao Wang ${ }^{3}$, Fan Yang ${ }^{2}$, Qiufei Wang ${ }^{3}$, Li Zhou ${ }^{2}$, \\ Lei Yang ${ }^{1}$, Shaoqing $\mathrm{Ju}^{4}$ \\ ${ }^{1}$ Department of Medical Oncology, Affiliated Tumor Hospital of Nantong University, Nantong, China; ${ }^{2}$ Medical School of Nantong University, \\ Nantong, China; ${ }^{3}$ Department of Orthopaedics, Affiliated Hospital of Nantong University, Nantong, China; ${ }^{4}$ Department of Laboratory Medicine, \\ Affiliated Hospital of Nantong University, Nantong, China \\ Contributions: (I) Conception and design: S Ju; (II) Administrative support: L Yang; (III) Provision of study materials or patients: L Yang, C Peng; (IV) \\ Collection and assembly of data: S Wang, F Yang, Q Wang; (V) Data analysis and interpretation: Y Huang, J Tang, L Zhou; (VI) Manuscript writing: \\ All authors; (VII) Final approval of manuscript: All authors. \\ \#These authors contributed equally to this work. \\ Correspondence to: Shaoqing Ju. Department of Laboratory Medicine, Affiliated Hospital of Nantong University, 20 Xisi Road, Nantong 226001, \\ China. Email: jsq814@hotmail.com; Lei Yang. Department of Medical Oncology, Affiliated Tumor Hospital of Nantong University, Nantong \\ 226361, China. Email: leiyang.53@163.com.
}

Background: Heat shock protein A12B (HSPA12B) plays a considerable protective role for cells, tissues, and organs against various noxious conditions. However, the expression of HSPA12B in cancer biology remains controversial. This study aimed to investigate the expression of HSPA12B and its role in cell adhesion mediated drug resistance (CAM-DR) of non-Hodgkin's lymphoma (NHL).

Methods: In this study, the expression of HSPA12B in NHL was determined by immunohistochemical, and the effect of HSPA12B expression on the prognosis of NHL was analyzed by Kaplan-Meier curves. Then, the transfection technique was used to research the effect of HSPA12B in cell apoptosis. The most important was to study the expression changes of HSPA12B in the adhesion model and the effect of overexpression of HSPA12B on CAM-DR.

Results: We analyzed the relationship between the expression levels of HSPA12B and clinical parameters in NHL. The expression of HSPA12B was directly related to the different NHL variants. We overexpressed HSPA12B in 2 NHL cell lines and found a subsequent reduction in apoptosis. More specifically, we used an adhesion assay to demonstrate that HSPA12B expression was induced in NHL cells when they adhered to fibronectin (FN) or bone marrow stroma cells (BMSCs). Finally, it was revealed that HSPA12B overexpression enhances CAM-DR.

Conclusions: Our data suggest that HSPA12B may play a functional role in CAM-DR and is thus a potential novel target for NHL treatment.

Keywords: Non-Hodgkin's lymphoma (NHL); heat shock protein A12B (HSPA12B); immunohistochemistry; cell adhesion mediated drug resistance (CAM-DR)

Submitted Jul 30, 2021. Accepted for publication Sep 10, 2021.

doi: 10.21037/atm-21-4185

View this article at: https://dx.doi.org/10.21037/atm-21-4185

\section{Introduction}

Non-Hodgkin lymphomas (NHL) are lymphoproliferative malignancies that arise out of mature lymphocytes within secondary lymphoid organs with a variety of biologic and histologic subtypes (1). The presentation of NHL involves both disseminated and localized disease with spread to secondary sites and insensitivity to therapy, and is associated with an unfavourable prognosis (2). The 
incidence of NHL is increasing worldwide with significant variations in temporal and regional trends detected for individual NHL histological subtypes (3). Histological subtypes of NHL include diffuse large B-cell lymphoma (DLBCL), follicular lymphoma (FL), Burkitt lymphoma (BL), extranodal lymphoma of mucosa-associated lymphoid tissue (MALT), and nasal natural killer/T-cell lymphoma (NK/T) (4). These histological variances are strongly sensitive to chemotherapy, radiotherapy, and targeted therapy, however, the ultimate goal of targeting all NHL subtypes remains to be elucidated. Approximately 10-20\% of patients with NHL show improvement with additional high-dose chemotherapy following allogeneic hemopoietic stem cell transplantation, suggesting that treatment failure in lymphoma is largely due to multi-drug resistance (5).

Tumor cells are affected by their microenvironment through cell-cell interactions, including direct contact and the action of soluble factors (6). Mounting evidence shows that, indeed, tumor recidivate after drug stimulation is induced by the bone marrow microenvironment (7) composed growth factors, soluble cytokines, hormones and components of bone marrow stromal cells (BMSCs), and extracellular matrix proteins (8). Some evidence has shown that specific niches within the lymphoma tumor microenvironment provide a sanctuary for subpopulations of lymphoma cells via stromal-tumor cell interactions (9). Over the last decade, studies have shown that adhesion of NHL cells to components of the extracellular matrix [e.g., fibronectin $(\mathrm{FN})$ ], contributed to a novel but reversible drug resistance-termed cell adhesion-mediated drug resistance (CAM-DR) (10). However, the molecular mechanisms underlying lymphoma which contribute to increased survival during the tumor cell response to therapy remain to be elucidated. With this study, we aimed to show that blocking the interaction between tumor cells and stromal cells will be a novel therapeutic target for tumors, especially hematologic malignancies (11). Notably, the proliferation, apoptosis, migration, and invasion of tumor cells are regulated by the expression of oncogenes and tumor suppressor genes, which are usually involved in CAM-DR (12); therefore, CAM-DR could be a key therapeutic target for NHL.

Heat shock protein A12B (HSPA12B) is the youngest member of a newly described subfamily of proteins distantly related to the $70-\mathrm{kDa}$ family of the mammalian heat shock proteins (HSP70) family 2003 (13). HSPA12B expression is restricted to specific cell types, a feature not common to heat shock proteins, which highlights its importance to endothelial cells function. Indeed, studies have established that HSPA12B is necessary for angiogenesis and chemotherapy resistance, specifically in the processes of proliferation, adhesion, migration, and tube formation $(14,15)$. Recently, it was shown that HSPA12B can activate the phosphatidylinositol 3-kinase/protein kinase B (PI3K/Akt) signaling pathway, which was directly linked to protecting against endotoxin-induced cardiac dysfunction (16). Activation of the PI3K/Akt pathway has been demonstrated to negatively regulate nuclear factor kappa-B (NF-кB) binding activity (17). Overexpression of HSPA12B attenuated the levels of $\mathrm{I}-\mathrm{kB} \alpha 16$. Previously published results from our lab have confirmed that HSPA12B expression can regulate neuronal apoptosis (18). Zappasodi et al. reported that the family of heat shock proteins, including HSP105 and HSPPC-96, were novel NHL therapeutic targets $(1,19)$. It was also shown that HSPA12B can stimulate lung cancer growth through Cox2-dependent mechanism (20), affect the apoptosis by regulating the B-cell lymphoma 2 (Bcl-2) family (21), and induce cisplatin resistance in non-small cell lung cancer by regulating the PI3K/Akt/NF- $\mathrm{kB}$ signal pathway (22), which is a potential therapeutic target for the treatment of lung cancer. Similar results have been echoed in findings where the heat shock protein families directly affect the overall survival of different types of breast cancer (23). However, the role of HSPA12B and its biological function in NHL have yet to be investigated.

In our study, we aimed to show the expression of HSPA12B in NHL variants and determine the correlation between the expression of HSPA12B and the pathological and clinical features in NHL. This study is the first to study the role of HSPA12B in hematopoietic tumors, especially in CAM-DR. HSPA12B can be used not only as a predictor of the prognosis of NHL, but also as a predictor of therapeutic effect, and it is more hopeful that combined therapy can reverse drug resistance. Our study was the first to report that HSPA12B affects the CAM-DR of NHL and may be of great value for future therapies.

We present the following article in accordance with the MDAR reporting checklist (available at https://dx.doi. org/10.21037/atm-21-4185).

\section{Methods}

\section{Patients and lymph node samples}

Lymph node samples were collected from 19 patients with reactive lymphadenopathy (RL), 16 patients with follicular 
lymphoma (FL), 37 patients with DLBCL, 28 mucosaassociated lymphoid tissue B cell lymphomas (MALT), and 15 nasal natural killers (NK)/T-cell lymphoma (NK/T). Samples were then fixed in formalin and embedded in paraffin. All samples were collected at the Affiliated Tumor Hospital of Nantong University, from 1 January 2000, to 1 April 2005. Histopathological and clinical diagnoses were in keeping with World Health Organization (WHO) criteria. In this study, informed consent was provided by all individuals. The collections of tissue samples of patients were approved by the Ethics Committee of the Affiliated Tumor Hospital of Nantong University (approval No. 2000-157). The study was conducted in accordance with the Declaration of Helsinki (as revised in 2013).

\section{Immunobistochemical and immunofluorescence labeling of HSPA12B}

Immunohistochemistry (IHC) was performed as previously described (24). The intensity of the IHC signal in each tumor section was assessed semiquantitatively using the following scale: as strong [3], moderate [2], weak [1], or negative [0]; semiquantitatively using the following scale: negative $[0]<1 \%$ of cells, weak [1] $=1-24 \%$ of cells, moderate [2] $=25-50 \%$ of cells, and strong [3] $>50 \%$ of cells, followed by multiplication of these values. This resulted in an overall HSPA12B IHC score of $0,1,2,3,4$, 6 , or 9 . The expression of HSPA12B was considered high when scores were $\geq 4$, and low when scores were $\leq 3$.

Daudi cells were cultured in FN coated flasks for 48 h. Next, cells were fixed with $8 \%$ paraformaldehyde $(1 \mathrm{~h})$, permeabilized with $0.1 \%$ Triton $\mathrm{X}-100(15 \mathrm{~min})$, and blocked for non-specific binding in phosphate-buffered saline (PBS), $1 \%$ milk for $2 \mathrm{~h}$. Cells were respectively labeled with anti-HSPA12B at $4{ }^{\circ} \mathrm{C}$ overnight. The cells were washed with PBS 3 times for 10 min each time, followed by labeling with secondary antibody (CY3Donkey-anti-Goat, 1:800, Jackson Laboratory, Bar Harbor, ME, USA) in the darkroom and incubated for another $2-3 \mathrm{~h}$ at room temperature. The DNA was stained by incubating the cells with Hoechst 33,342 dye $(1 \mu \mathrm{g} / \mathrm{mL}, 10 \mathrm{~min})$ after labeling with secondary antibodies. Cells with nuclear versus cytoplasmic HSPA12B were enumerated visually on a Leica fluorescence confocal (Wetzlar, Germany).

\section{Western blot analysis and coimmunoprecipitation}

Western blot was performed as described previously (24).
Bound proteins were separated by sodium dodecyl sulfate-polyacrylamide gel electrophoresis (SDS-PAGE), transferred to a nitrocellulose membrane (Schleicher and Schuell Bioscience, Keene, NH, USA), and subjected to western blot analysis with antibodies against HSPA12B (1:1,000; Santa Cruz Biotechnology, Inc., Dallas, TX, USA, sc-393635); Bcl-2 (1:500, Santa Cruz Biotechnology, sc7382), Bax (1:500, Santa Cruz Biotechnology, sc-7480), P65 (1:500, Cell Signaling Technology, Danvers, MA USA, cat.3033S), P50 (1:1,000, Cell Signaling, cat.3035S), PI3K (1:1,000, Santa Cruz Biotechnology, sc-365290), and glyceraldehyde 3-phosphate dehydrogenase (GAPDH; 1:1,000, Santa Cruz Biotechnology, sc-365062) overnight at $4{ }^{\circ} \mathrm{C}$. Membranes were washed 3 times with Tris-buffered saline Tween 20 (TBST) and incubated with horseradish peroxidase-conjugated secondary antibody for $2 \mathrm{~h}$. For immunoprecipitation assays, cells were collected in PBS and lysed using radioimmunoprecipitation assay (RIPA) buffer. We used G Sepharose beads to clear the lysate from protein prior to incubation with a specific antibody bound to protein A/G Sepharose beads (Thermo Fisher Scientific, Waltham, MA, USA, cat.88802) for $12 \mathrm{~h}$ at $4{ }^{\circ} \mathrm{C}$. Precipitated immune-complexes were washed with RIPA buffer 4 times, eluted by boiling in $2 \times$ SDS sample buffer, resolved by SDS-PAGE gel, and analyzed by immunoblotting as previously reported. The signals were visualized using an electrochemiluminescence (ECL) detection system. The results were derived from at least 3 independent experiments.

\section{Cell culture and transfection}

For this study, our lab received the Daudi human NHL cell line and the human bone marrow stromal cells (BMSC) line HS-5 as a gift from the Jiangsu Institute of Hematology, China. The human NHL cell line OCI-LY8 was obtained from the Department of Oncology, Affiliated Cancer Hospital of Fudan University. The NHL cells were cultured in suspension in Roswell Park Memorial Institute (RPMI) 1640 media (R8758, Sigma-Aldrich, Rehovot, Israel) and HS-5 cells were cultured in F12 (N6658, Sigma-Aldrich, Rehovot) supplemented with $10 \%$ fetal bovine serum at $37{ }^{\circ} \mathrm{C}$ and with $5 \% \mathrm{CO}_{2}$. The OCI-LY8 and Daudi cells were transfected with GFP-HSPA12B or green fluorescent protein (GFP)-control using lentiviral particles (NO. KL0009L5, Genechem, Shanghai, China). Lentiviruses were then added into cell suspensions in the presence of $8 \mu \mathrm{g} / \mathrm{mL}$ polybrene, and transduced for $72 \mathrm{~h}$. Transduction 
efficiencies were observed by GFP expression using a fluorescence microscope.

\section{Co-culture of NHL cells with stromal cells and adhesion assay}

For co-culture experiments, stromal cells HS-5 were seeded in plates to near confluence. After stromal cells were washed once with fresh medium, NHL cells were added to the plates. After $24 \mathrm{~h}$, the adherent cells were harvested for downstream experiments, with the HS-5 monolayer kept intact.

To determine the specificity of adhesion of Daudi and OCI-LY8 cells to HS-5 cells or FN we used a nest assay (Sigma-Aldrich, Rehovot). We coated 96-well plates with either $50 \mu \mathrm{L}(40 \mathrm{mg} / \mathrm{mL})$ of FN or monolayer HS-5 cells at $37{ }^{\circ} \mathrm{C}$ overnight. Cells were resuspended in serumfree RPMI 1640 media, at a density of $1 \times 10^{6} / \mathrm{mL}$. Before adhesion, lymphocytes were incubated with calcein (Santa Cruz Biotechnology, sc-202090) for 30 min according to the manufacturer's protocol. After 2-4 h of adhesion, the non-adherent cells were washed off twice with $1 \mathrm{~mL}$ of PBS and the absorbance was measured using an automated plate reader at a wavelength of $490 \mathrm{~nm}$ at different time points.

\section{Cell viability assay}

Cell counting kit-8 (CCK-8) (Dojindo Molecular Technologies, Gaithersburg, MD, USA, cat.CK-04) was employed to assess cell viability. Lymphocytes were plated on a 96 well plate at the density of $10^{5}$ cells/well. Cells were then treated with doxorubicin or mitoxantrone at different time points. The CCK- 8 reagents were added to each well according to the manufacturer's protocol followed by an additional $1 \mathrm{~h}$ of incubation at $37{ }^{\circ} \mathrm{C}$ and $5 \% \mathrm{CO}_{2}$. The absorbance was measured at $450 \mathrm{~nm}$ using an automated plate reader. These experiments were repeated in triplicate.

\section{Flow cytometry-based annexin V/propidium iodide staining}

Flow cytometry was performed to investigate the degree of apoptosis and necrosis of Daudi and OCI-LY8 cell lines. Briefly, samples from each experimental group were collected, washed with ice-cold PBS, stained with propidium iodide (PI) and ApoScreen Annexin V kit (Southern Biotechnology, Birmingham, AL, USA) according to the manufacturer's instructions prior to FACScan flow cytometry (BD FACSAriaII, Becton, Dickinson and Co., Franklin Lakes, NJ, USA).

\section{Statistical analysis}

Statistical analysis was performed using the statistical software package PASW 18 (IBM Corp., Chicago, IL, USA). Chi-square $\left(\chi^{2}\right)$ test was used to determine associations between HSPA12B expression and any clinicopathological features. For the analysis of survival data, Kaplan-Meier curves were constructed and the log-rank test was performed. Multivariate analysis was performed using Cox's proportional hazards model, with $\mathrm{P}<0.05$ considered statistically significant. Data were presented as mean \pm standard deviations (SD) of 3 independent experiments.

\section{Results}

\section{Expression of HSPA12B correlates with the different varieties of NHL directly}

To investigate the potential role of HSPA12B in NHL subtypes, we first analyzed the expression levels of HSPA12B in NHL samples and reactive lymphadenopathy (RL) tissue samples using IHC. A PBS-based control was used to evaluate the specificity of IHC signaling (Figure $1 A, 1 B$ ). As shown in Figure $1 C-1 L$, the expression of HSPA12B appeared to be much lower in NHL samples (Figure 1C,17) than it was in nontumor tissue (Figure $1 K, 1 L$ ). Moreover, the expression level was remarkably higher in indolent lymphomas (Figure 1G-17) than that in aggressive NHL (Figure 1C-17). We next determined the HSPA12B expression with high-risk clinical parameters in NHL. We detected that the lymphoma cases present low expressed levels of HSPA12B at $41.46 \%$ in B lymphoma samples (34/82), $12.50 \%$ in T lymphoma samples (3/24), but $95.65 \%$ in normal lymph tissue (22/23) (Table S1). We also detected a highly significant difference of HSPA12B expression between NHL and normal lymph tissue $(\mathrm{P}<0.05)$, indicating the preferential expression pattern of HSPA12B in human lymphoma tissues.

\section{Correlation between expression of HSPA12B and survival rates in patients with NHL}

Survival analysis was restricted to 106 patients with available complete follow-up data and results for HSPA12B expression levels. We assessed 2 aggressive subtypes of NHL, namely DLBCL and NK/T, which represent the B and $\mathrm{T}$ cell lineage in China. Among aggressive subtypes of NHL, DLBCL is the most prevalent and represents $\sim 30 \%$ of all NHL diagnoses in adults (25). Due to various types of samples, we selected the 2 most representative 



Figure 1 HSPA12B expression and distribution was examined in NHL or non-tumor tissue by IHC. IHC was performed to detect HSPA12B expression in DLBCL (C, 20×; D, 40×), NK/T (E, 20×; F, 40×), MALT (G, 20×; H, 40×), FL (I, 20×; J, 40×), RL (K, 20×; L, 40×) and negative control (A, 20×; B, 40x). The experiment details were described in "Materials and methods". NHL, non-Hodgkin's lymphoma; IHC, immunohistochemistry; DLBCL, diffuse large B-cell lymphoma. 

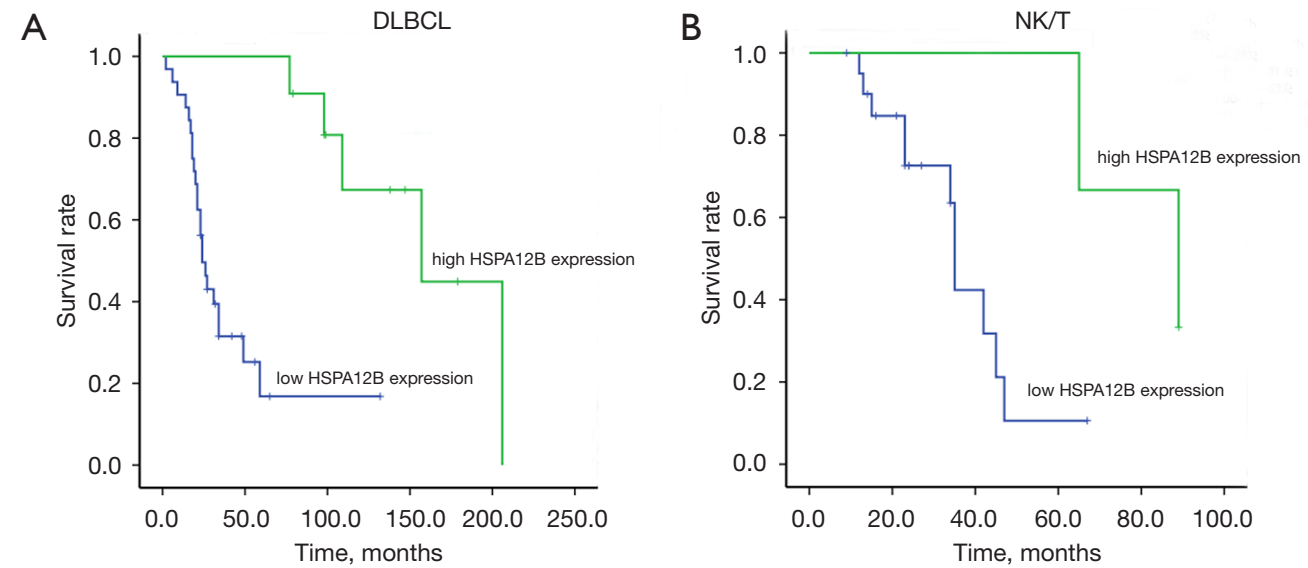

Figure 2 Cumulative survival curves according to HSPA12B expression. Based on the score of HSPA12B, patients were divided into high HSPA12B expressers (score $\geq 4$ ) and low HSPA12B expressers (score $\leq 3$ ). Patients in the low-expression HSPA12B group had significantly shorter overall survival (A, in DLBCL; B, in NK/T). DLBCL, diffuse large B-cell lymphoma; NK/T, nasal natural killers (NK)/T-cell lymphoma.

of NHL for analysis. Kaplan-Meier analysis showed a significant association between patients with low expression of HSPA12B and short overall survival in both DLBCL (Figure $2 A$ ) and NK/T (Figure $2 B)(\mathrm{P}<0.05)$. According to the results of histochemical expression and statistical analysis, the low expression of HSPA12B can indicate that NHL is highly invasive and can be used as a predictor of poor prognosis of NHL. In the future clinical application, targeted drugs or reagents for HSPA12B over-expression will be constructed to increase the expression of HSPA12B in lymphoma, especially in patients with DLBCL and NK/T, and improve progression time and survival time of patients. In other relatively rare subspecies, the number of samples can be increased later to verify the effect of HSPA12B expression on their prognosis.

\section{Regulation of cell apoptosis by HSPA12B in NHL cells}

In the next set of studies, we focused on the effect of HSPA12b on cell apoptosis in NHL because HSPA12b was found to be closely related to tumor apoptosis in lung cancer, and anti-apoptosis was a key factor in CAMDR. To further study the potential effects of HSPA12B on NHL cells apoptosis, OCI-LY8 and Daudi cells were transfected with GFP-HSPA12B or GFP-control for $48 \mathrm{~h}$, and the transfection efficiency was assessed (Figure $3 A$ ). The viability rates of cells transfected with GFP- HSPA12B in doxorubicin $(0.5 \mu \mathrm{mol} / \mathrm{L})$ were significantly accelerated (Figure 3B). This data was consistent with the up-regulation of the cell pro-apoptotic protein marker $\mathrm{Bcl}-2$ and the down-regulation of anti-apoptotic marker Bax (Figure 3A). In addition, we used a flow cytometry assay to determine the cell apoptosis of over-expression of HSPA12B by assessing the levels of annexin $\mathrm{V}$ positive cells. To differentiate necrotic cells from live and apoptotic ones, we stained with PI, a red fluorescent dye that binds tightly to the nucleic acids in necrotic cells. We found that over-expression of HSPA12B led to significant decreases in Annexin V-positive cells (Figure 3C). Quantification analysis is illustrated in Figure $3 D$, the results were consistent with the study on the anti-apoptosis role of HSPA12B. Our data were similar to results in lung cancer. In NHL, HSPA12b is also involved in cell apoptosis and was an anti-apoptotic factor.

\section{The expression of HSPA12B is associated with cell adhesion in NHL cells}

It has been reported that adhesion of NHL cells to FN or stromal cells results in CAM-DR, which relates to cell apoptosis $(10,26)$. Therefore, we inferred that HSPA12B might be involved in NHL's CAM-DR. To verify these results, we performed a cell adhesion assay which linked cell adhesion to the expression of HSPA12B. When Daudi and OCI-LY8 cell lines adhered to FN or HS-5 cells, they exhibited increased expression levels of HSPA12B, compared with cells grown in suspension (Figure 4A,4B). Confocal imaging also revealed that HSPA12B accumulated predominantly in the cytoplasm when the cell adhered 
A
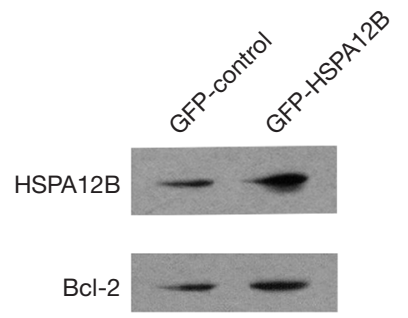

Bax

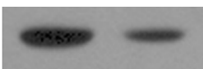

GAPDH

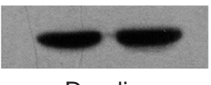

Daudi

C
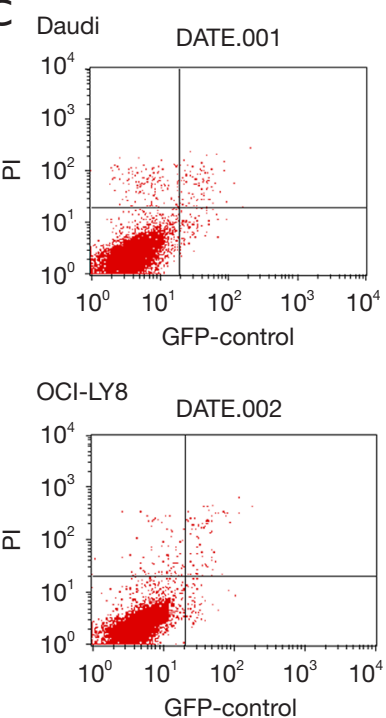
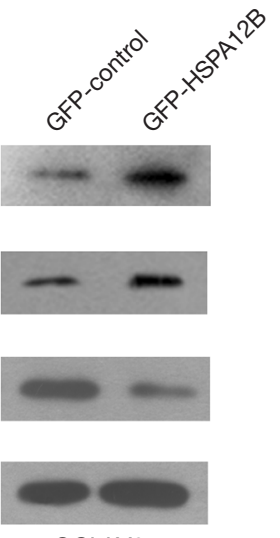

OCI-LY8
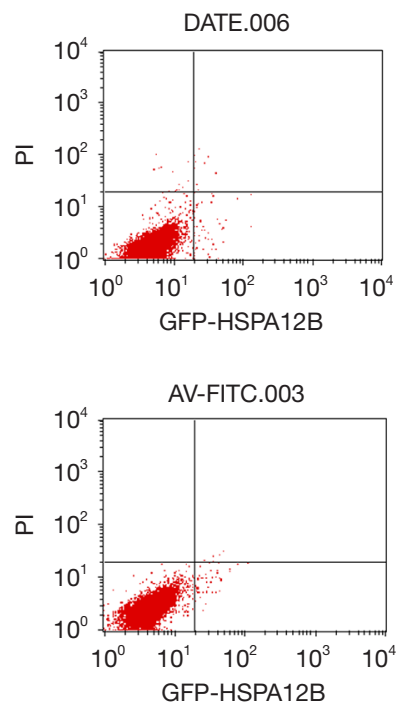

B

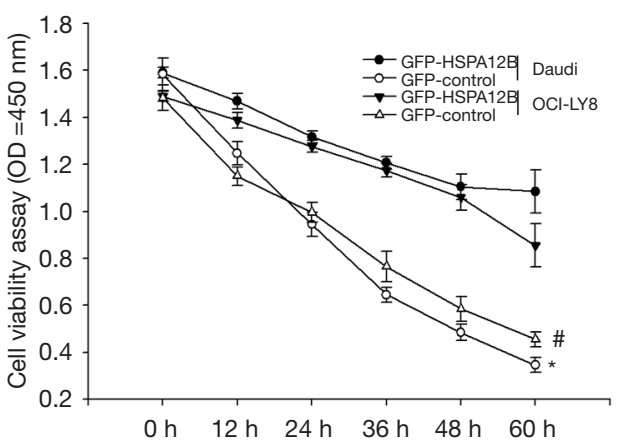

$\mathrm{D}$

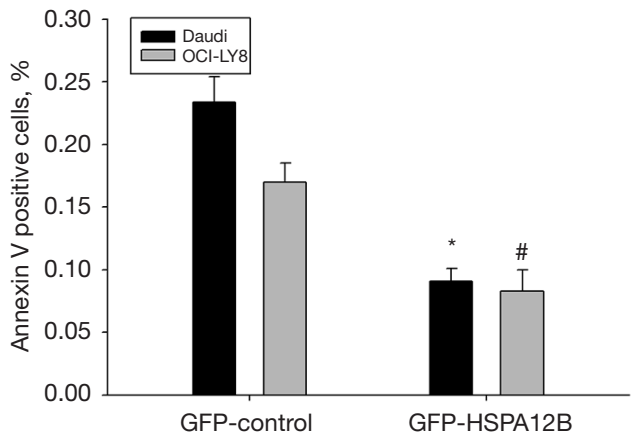

Figure 3 Over-expression of HSPA12B reduced the NHL cell apoptosis. (A) A representative western blot image showed the expression of HSPA12B, Bcl-2 and Bax in GFP-control and GFP-HSPA12B treated cells, respectively. GAPDH was used as the internal control for western blot analysis, respectively. (B) The comparison in cell vitality curves between the cells transfected with GFP-control and GFPHSPA12B with doxorubicin $(0.5 \mu \mathrm{mol} / \mathrm{L})$ in suspension, as measured by CCK-8 assay $\left(^{*},{ }^{*}: \mathrm{P}<0.05\right)$. (C) Flow cytometry assay showing that GFP-HSPA12B decreased the number of apoptotic cells. In the 4 fields of the original images from the flow cytometry-based study, the number of the dots indicates the number of annexin V-/PI- (bottom left field), annexin V+/PI- (bottom right field), annexin V-/PI+ (top left field), and annexin V+/PI+ cells (top right field). (D) Quantification of the results from the flow cytometry-based study ( ${ }^{*}$, ": $\mathrm{P}<0.05$, compared with the control group). NHL, non-Hodgkin's lymphoma; GFP, green fluorescent protein; GAPDH, glyceraldehyde 3-phosphate dehydrogenase; CCK-8, Cell Counting Kit-8; PI, propidium iodide.

to $\mathrm{FN}$, while in suspension conditions, HSPA12B was mainly concentrated in the nucleus (Figure 4C). These results suggested that nuclear export may be triggered by cell adhesion to FN. Cell adhesion assay revealed that the adhesion rate was significantly increased after overexpression of HSPA12B (Figure 4D). Taken together, these data suggested that adhesion to $\mathrm{FN}$ or bone marrow stroma up-regulated HSPA12B expression, which in turn increased cell adhesion. To our knowledge, this was the first time that the existence of an adhesion-dependent regulation of HSPA12B was reported.

\section{HSPA12B overexpression generated CAM-DR}

The previous results showed that the adhesion of NHL cells and the expression of HSPA12b promoted each other, 
A

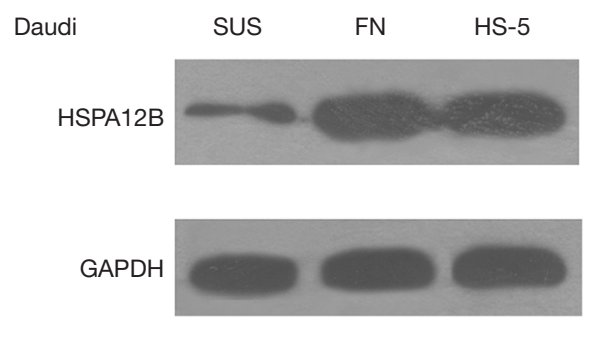

OCI-LY8

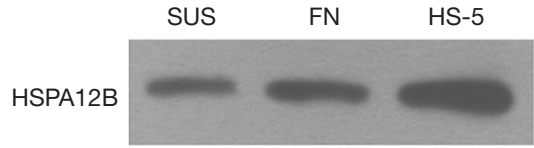

GAPDH

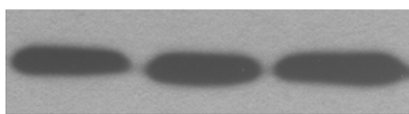

C

HSPA12B
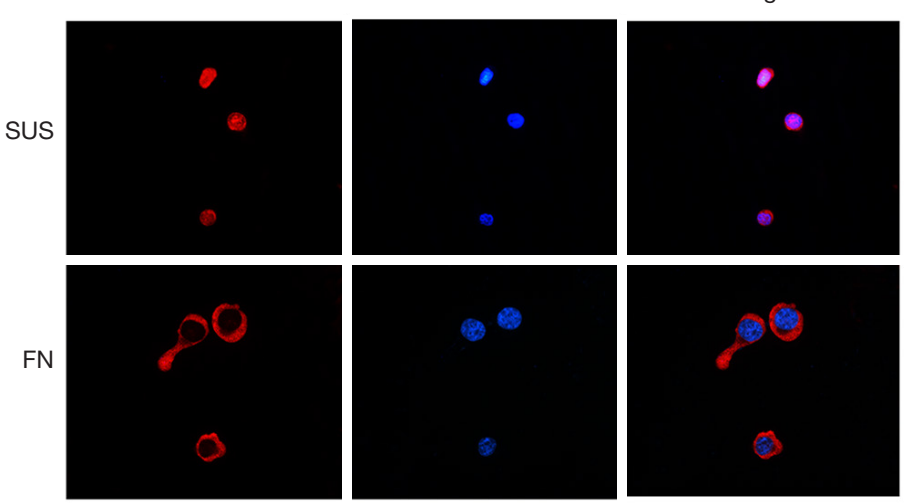

B
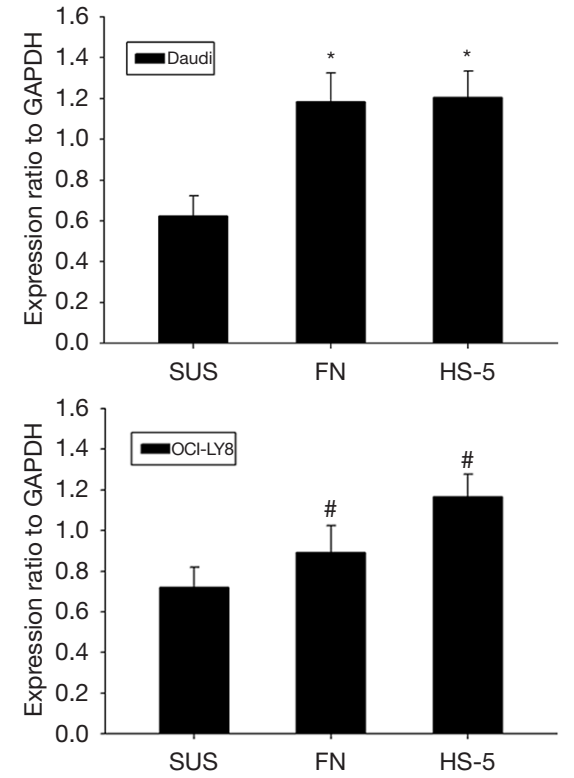

D

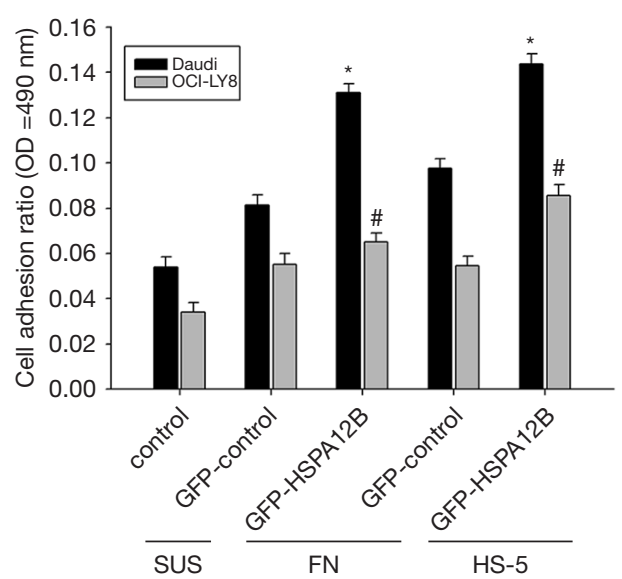

Figure 4 Adhesion of NHL cells to FN or HS-5 cells induces HSPA12B expression. (A) Daudi and OCI-LY8 cells have adhered to FN and HS-5 cells for $24 \mathrm{~h}$ or cultured in suspension (SUS) as described in "Materials and methods". The western blot image showed the expression of HSPA12B in three different kinds of conditions. GAPDH was used as the loading control. (B) The bar chart demonstrates the ratio of HSPA12B expression to GAPDH by densitometry. The data are mean \pm SD of 3 independent experiments (*, \#: P<0.05 compared with the SUS). (C) Adhered Daudi cells and suspicion cells were then fixed with paraformaldehyde. The cellular localization of HSPA12B was detected by immunofluorescence staining. Original magnification $\times 400$. (D) The cell adhesion rate of Daudi and OCI-LY8 cells transfected with GFP-control or GFP-HSPA12B was determined as described above. A representative of three independent experiments was shown $\left({ }^{*},{ }^{*}\right.$ : $\mathrm{P}<0.05$ compared with the control group). FN, fibronectin; GAPDH, glyceraldehyde 3-phosphate dehydrogenase; SD, standard deviation.

and the increase of cell adhesion was the key to drug insensitivity, that is, drug resistance and treatment failure. Therefore, this section reports on the relationship between HSPA12b and drug resistance. To determine if HSPA12B could desensitize NHL cells to the chemotherapeutic agent, Daudi (Figure 5A) and OCI-LY8 (Figure 5B) cells were treated with doxorubicin $(1 \mu \mathrm{mol} / \mathrm{L})$ or mitoxantrone
( $2 \mu \mathrm{mol} / \mathrm{L})$. Overexpression of HSPA12B decreased doxorubicin or mitoxantrone induced apoptosis when cultured under different conditions. These data suggest that HSPA12B is crucial for the survival of adherent NHL cells, and that down-regulation of HSPA12B may contribute to the apoptosis of drug-resistant cells and therefore reverse CAM-DR. According to our previous reports, there are 
A

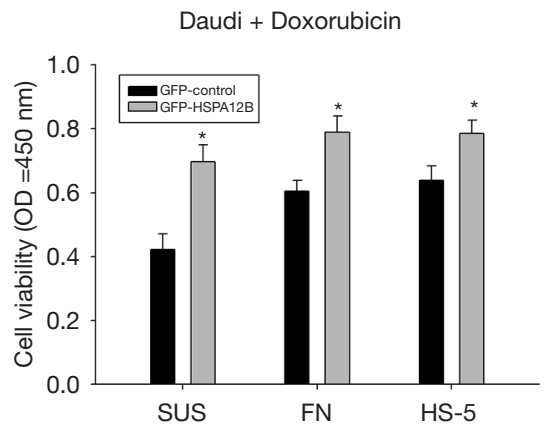

B

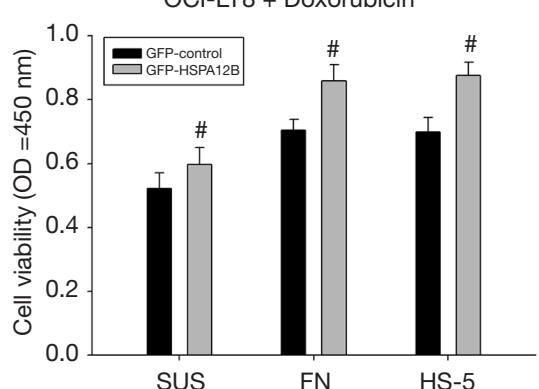

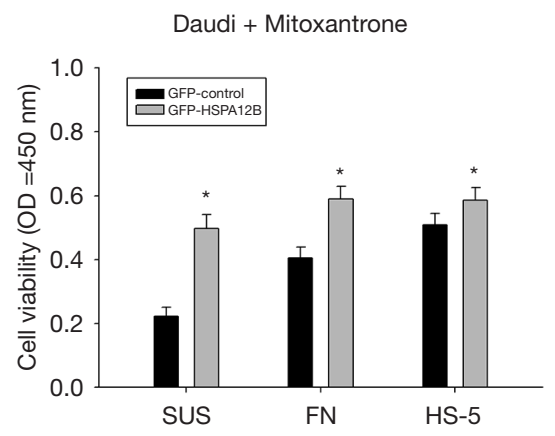

OCI-LY8 + Mitoxantrone

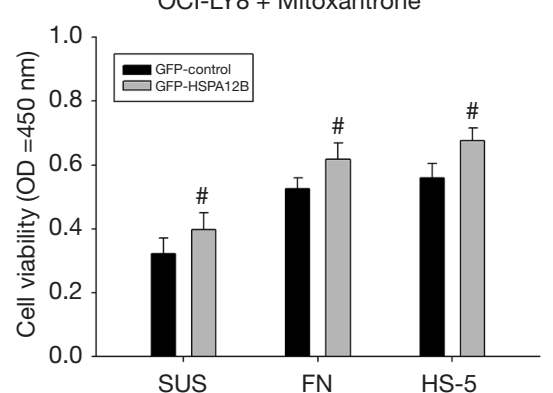

$E$

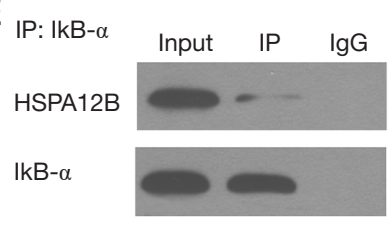

HSPA12B

p65

Input IP IgG

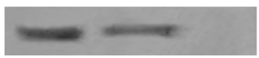

IP: p50

HSPA12B

p50

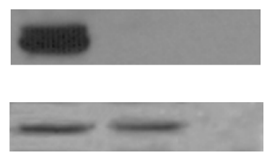

Figure 5 HSPA12B induced CAM-DR in Daudi and OCI-LY8 cells. (A) Daudi cells were transfected with GFP-control and GFPHSPA12B and then treated with doxorubicin (left) or mitoxantrone (right) adhering to FN, or HS-5 cells or culturing in suspension for 72 h. CCK-8 assay was performed following the addition of doxorubicin for evaluation of cell viability. (B) OCI-LY8 cells were treated with the same conditions. Data shown are representative of 3 independent experiments (*, *: $\mathrm{P}<0.05$, compared with the control group). (C) A representative western blot image showed the expression of p65, p50, and PI3K in FN, HS-5, or SUS. (D) The bar chart demonstrates the ratio of p65, p50, and PI3K expression to GAPDH by densitometry. The data are mean $\pm \mathrm{SD}$ of three independent experiments ${ }^{*}$, \#: $\mathrm{P}<0.05$, compared with the SUS). (E) Daudi cell lysates were prepared and subjected to immunoprecipitation (IP) with antibodies to IкB- $\alpha$, p65, and p50. The resulting precipitates were subjected to IB analysis with antibodies to HSPA12B. Details of the experiments are given in "Methods". CAM-DR, cell adhesion mediated drug resistance; CCK-8, Cell Counting Kit-8; FN, fibronectin; GAPDH, glyceraldehyde 3-phosphate dehydrogenase; SD, standard deviation; IB, immunoblot.

many signal pathways involved in CAM-DR, including PI3K/AKT, NF-kB, BCL-2/BCL-XL pathway, etc. (27). The pathways of $\mathrm{NF}-\kappa \mathrm{B}$ and PI3K/Akt have been known as the important signaling pathways to promote the survival of hematological malignancies cells in CAM-DR
$(10,28,29)$. It has been found that PI3K/AKT is involved in the occurrence and development of CAM-DR in multiple myeloma (MM) and NHL. CAM-DR is associated with the

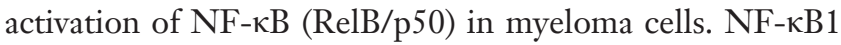
(p50) homodimers contribute to transcription of the Bcl- 
2 oncogene (30). After Daudi cells adhered to FN or HS-5 cells, the protein levels of p65 and p50 increased, which verified the positive role of NF- $\kappa \mathrm{B}$ signaling pathways (Figure 5C,5D). This was not coupled with a change in PI3K protein expression. Thus, we assumed that HSPA12B affected the CAM-DR by regulating the activity of NF$\kappa \mathrm{B}$ signaling pathways. To assess that hypothesis, we used immunoprecipitation to show that HSPA12B could interact with IкB- $\alpha$ in Daudi cells (Figure $5 E$ ). In contrast, we did not find an interaction between HSPA12B and p65 or p50 (Figure $5 E$ ). Collectively, the results from this study illustrated that HSPA12B regulates the CAM-DR in NHL via the NF- $\mathrm{BB}$ signaling pathway. In the future, the research on PI3K inhibitors will also be the direction of our research on CAM-DR. Our results provide novel insight to understanding the mechanism underlying the drug resistance of NHLs, which paves the way for a new therapeutic approach in targeting this molecule.

\section{Discussion}

With the development of an aging society, cancer has become a killer endangering human health. In recent years, the incidence, prevalence, and fatality rate of malignant tumors have shown an obvious upward trend. At present, despite the continuous improvement of various therapeutic techniques, especially the combined use of conventional drugs combined with monoclonal antibodies and immunotherapy, the overall prognosis of tumors has been significantly improved, but malignant tumors in the middle and advanced stages still cannot be completely cured. Although the origin and mechanism of lymphatic hematopoietic malignancies and solid tumors are different, as well as their response to treatment and prognosis, resistance to chemotherapy drugs will occur in both of them, which will lead to the progression and recurrence of the disease. Existing literature suggests that CAM-DR induced by the tumor microenvironment plays a non-negligible role in the drug resistance mechanism of hematological malignancies, especially in NHL and MM $(7,31)$.

The Hsp70s constitute one group of the heat shock protein superfamily, classified according to their molecular mass: Hsp10, Hsp40, Hsp60, Hsp70, Hsp90, and Hsp110. It has been shown that Hsp70s are capable of protecting cells and tissues from ischemic injury (32,33). In particular, HSPA12B contains an atypical HSP70 ATPase domain which is a key component required for cell adhesion and cell migration $(13,14)$. In recent years several studies have investigated the activation of the heat shock response (HSR), especially HSP70 levels, which are involved in regulating angiogenesis and apoptosis in several human disease models $(14,34)$. Previous studies have demonstrated that these angiogenic and apoptotic effects were associated with tumorigenesis and chemotherapy efficacy (35-37). The disease NHL has a high rate of angiogenesis which ultimately leads to chemotherapy resistance (38-40). Lymphoma cells migrate to bone marrow or secondary lymphoid organs, suggesting that the tumor microenvironment may provide the required components for the survival of tumor cells (7). Using IHC, we detected HSPA12B expression in both reactive lymphoid tissues and different lymphomas, including DLBCL, FL, MALT, and NK/T-cell lymphoma. Multivariate analysis revealed that the expression of HSPA12B was associated with high-risk clinical parameters and it was identified as an independent prognostic factor for NHL. This data clearly identified that HSPA12B may be a significant factor for detecting a poor prognostic group of NHL.

In our present study, western blot analysis documented an increased expression of HSPA12B in drug-mediated NHL cell apoptosis. Inhibition of apoptosis is a key mechanism used to enable cancer cells to proliferate and grow unabated. Therefore, the results from our study suggested that HSPA12B is involved in cell apoptosis after being incubated with chemotherapeutics (doxorubicin or mitoxantrone), but the exact role of HSPA12B has yet to be elucidated. The result of the expression of HSPA12B in apoptosis was consistent with that in the nervous system and inflammatory response $(14,34,41)$. Our paper, for the first time, demonstrated the expression of HSPA12B and its biological function in human malignant neoplasm, especially in hematological malignancy.

Chemotherapy drug resistance is the main cause of tumor recurrence and incurability, and the non-negligible cause of chemotherapy drug resistance is the effect of the tumor microenvironment. The tumor microenvironment mainly includes stromal cells, soluble cytokines, and extracellular matrix proteins, etc. (27). The environment-mediated drug resistance (EM-DR) is the synergistic effect of soluble factor mediated-drug resistance (SM-DR) and CAM-DR, which mainly mediates the development of the initial drug resistance (11). Among them, CAM-DR is an important cause of incurable tumors. This article focused on the role of HSPA12B in the clinical significance of the tumor microenvironment. Through increased understanding of these interactions, new, specific, and more rational therapies 
can be developed.

We found that after $12 \mathrm{~h}$ of being adherent to $\mathrm{FN}$ or HS-5 cells, the levels of HSPA12B increased significantly and there was also a change of expression location within the cell. To further assess the role of HSPA12B in adhesion, we used GFP-HSPA12B to overexpress the protein. These results demonstrated that adhesion to $\mathrm{FN}$ or BMSCs induced HSPA12B expression, which in turn facilitated cell adhesion. Collectively these experiments showed that HSPA12B plays an important role in NHL cell adhesion in vitro. It is possible that HSPA12B may associate with CAM-DR to communicate its effects on cell adhesion. To demonstrate this hypothesis, we established an in vitro model of CAM-DR. Overexpression of HSPA12B decreased doxorubicin or mitoxantrone-specific induced apoptosis when cultured under different conditions. Adhesion between tumor cells and stromal cells activates various signaling pathways such as those of PI3K/AKT, $\mathrm{NF}-\kappa \mathrm{B}$, and Ras/Raf/MEK/MAPK, which ultimately leads to a high survival rate and drug resistance of tumor cells (42). Some signaling pathways, such as Janus-activated kinase signal transduction activator of transcription (JAK/STAT) and Wnt3/RhoA/ROCK, have been reported to be related to apoptosis of tumor cells (42). Under the same conditions, the protein levels of $\mathrm{p} 65$ and $\mathrm{p} 50$ increased, which verified the positive role of $\mathrm{NF}-\mathrm{\kappa B}$ signaling pathways. Previous studies suggested that inhibition of NF- $\mathrm{KB}$ prevents $\mathrm{p} 65$ subunit from entering the nucleus, which downregulates anti-apoptotic protein targets of $\mathrm{p} 65$ such as IкB- $\alpha$ activating the downstream mitochondrial apoptotic pathway (43). Similarly, we also investigated the PI3K/AKT signaling pathway and found that targeting of this survival pathway did not affect cell apoptosis, however, we did find that there was cross-talk between the NF- $\kappa \mathrm{B}$ and PI3K/AKT signaling pathways (43). IкB- $\alpha$ is an important member of NF- $\kappa \mathrm{B}$ signaling pathway, which mainly regulates the activation and transcription of NF- $\mathrm{KB}$ and participates in the occurrence and development of many diseases (44). The NF- $\kappa \mathrm{B}$ signal pathway is the key pathway in CAMDR. In this study, it is found that there is a regulatory effect between HSPA12B and IкB- $\alpha$. It is predicted that HSPA12B may be involved in the regulation of CAM-DR through $\mathrm{NF}-\kappa \mathrm{B}$ signal pathway, and the specific mechanism needs to be further confirmed. In this paper, we also detected the markers of the corresponding pathway, such as figure $3 \mathrm{~A}$ and $5 \mathrm{E}$, but there is no further research on PI3K/AKT or $\mathrm{NF}-\kappa \mathrm{B}$. In the future, the research on PI3K inhibitors will also be the direction of our research on CAM-DR. These findings provide an important clue to clarify the molecular mechanisms underlying CAM-DR. Further research on the pathogenesis and related signaling pathways of CAM-DR can provide the basis for clinical treatment and prognosis guidance.

In summary, we detected the different HSPA12B expressions in CAM-DR in NHL for the first time. Our data clearly demonstrates that HSPA12B plays a functional role in drug resistance of chemotherapy in NHL. Future studies in our lab will include further investigation of the mechanism of HSPA12B involved in CAM-DR for NHL. Our results may provide the way for a novel therapeutic target to approach this molecule.

\section{Acknowledgments}

Funding: This project was supported by grants from the National Natural Science Foundation of China (81600158, 81871720, and 82072363) and the National Funds for Distinguished Young Scientists of Nantong City (WQ2016057).

\section{Footnote}

Reporting Checklist: The authors have completed the MDAR reporting checklist. Available at https://dx.doi. org/10.21037/atm-21-4185

Data Sharing Statement: Available at https://dx.doi. org/10.21037/atm-21-4185

Conflicts of Interest: All authors have completed the ICMJE uniform disclosure form (available at https://dx.doi. org/10.21037/atm-21-4185). The authors have no conflicts of interest to declare.

Ethical Statement: The authors are accountable for all aspects of the work in ensuring that questions related to the accuracy or integrity of any part of the work are appropriately investigated and resolved. In this study, informed consent was provided by all individuals. The collections of tissue samples of patients were approved by the Ethics Committee of the Affiliated Tumor Hospital of Nantong University (approval No. 2000-157). The study was conducted in accordance with the Declaration of Helsinki (as revised in 2013).

Open Access Statement: This is an Open Access article 
distributed in accordance with the Creative Commons Attribution-NonCommercial-NoDerivs 4.0 International License (CC BY-NC-ND 4.0), which permits the noncommercial replication and distribution of the article with the strict proviso that no changes or edits are made and the original work is properly cited (including links to both the formal publication through the relevant DOI and the license). See: https://creativecommons.org/licenses/by-nc-nd/4.0/.

\section{References}

1. Zappasodi R, Bongarzone I, Ghedini GC, et al. Serological identification of HSP105 as a novel non-Hodgkin lymphoma therapeutic target. Blood 2011;118:4421-30.

2. Chao MP, Tang C, Pachynski RK, et al. Extranodal dissemination of non-Hodgkin lymphoma requires CD47 and is inhibited by anti-CD47 antibody therapy. Blood 2011;118:4890-901.

3. Laurini JA, Perry AM, Boilesen E, et al. Classification of non-Hodgkin lymphoma in Central and South America: a review of 1028 cases. Blood 2012;120:4795-801.

4. Hang Q, Fei M, Hou S, et al. Expression of Spy1 protein in human non-Hodgkin's lymphomas is correlated with phosphorylation of p27 Kip1 on Thr187 and cell proliferation. Med Oncol 2012;29:3504-14.

5. Yagi K, Yamamoto K, Umeda S, et al. Expression of multidrug resistance 1 gene in B-cell lymphomas: association with follicular dendritic cells. Histopathology 2013;62:414-20.

6. Di Marzo L, Desantis V, Solimando AG, et al. Microenvironment drug resistance in multiple myeloma: emerging new players. Oncotarget 2016;7:60698-711.

7. Burger JA, Ghia P, Rosenwald A, et al. The microenvironment in mature B-cell malignancies: a target for new treatment strategies. Blood 2009;114:3367-75.

8. Chen WC, Hu G, Hazlehurst LA. Contribution of the bone marrow stromal cells in mediating drug resistance in hematopoietic tumors. Curr Opin Pharmacol 2020;54:36-43.

9. Lwin T, Zhao X, Cheng F, et al. A microenvironmentmediated c-Myc/miR-548m/HDAC6 amplification loop in non-Hodgkin B cell lymphomas. J Clin Invest 2013;123:4612-26.

10. Mraz M, Zent CS, Church AK, et al. Bone marrow stromal cells protect lymphoma B-cells from rituximabinduced apoptosis and targeting integrin $\alpha-4-\beta-1$ (VLA4) with natalizumab can overcome this resistance. Br J Haematol 2011;155:53-64.
11. Kim HN, Ruan Y, Ogana H, et al. Cadherins, Selectins, and Integrins in CAM-DR in Leukemia. Front Oncol 2020;10:592733.

12. Huang Y, Huang X, Cheng C, et al. Elucidating the expression and function of Numbl during cell adhesionmediated drug resistance (CAM-DR) in multiple myeloma (MM). BMC Cancer 2019;19:1269.

13. Han Z, Truong QA, Park S, et al. Two Hsp70 family members expressed in atherosclerotic lesions. Proc Natl Acad Sci U S A 2003;100:1256-61.

14. Steagall RJ, Rusiñol AE, Truong QA, et al. HSPA12B is predominantly expressed in endothelial cells and required for angiogenesis. Arterioscler Thromb Vasc Biol 2006;26:2012-8.

15. Zouein FA, Kurdi M, Booz GW. HSPA12B and repairing the heart: beauty in simplicity. Cardiovasc Res 2013;99:587-9.

16. Zhou H, Qian J, Li C, et al. Attenuation of cardiac dysfunction by HSPA12B in endotoxin-induced sepsis in mice through a PI3K-dependent mechanism. Cardiovasc Res 2011;89:109-18.

17. Li XQ, Cao W, Li T, et al. Amlodipine inhibits TNF-alpha production and attenuates cardiac dysfunction induced by lipopolysaccharide involving PI3K/Akt pathway. Int Immunopharmacol 2009;9:1032-41.

18. Kang L, Zhang G, Yan Y, et al. The role of HSPA12B in regulating neuronal apoptosis. Neurochem Res 2013;38:311-20.

19. Oki Y, McLaughlin P, Fayad LE, et al. Experience with heat shock protein-peptide complex 96 vaccine therapy in patients with indolent non-Hodgkin lymphoma. Cancer 2007;109:77-83.

20. Ma H, Lu T, Zhang X, et al. HSPA12B: a novel facilitator of lung tumor growth. Oncotarget 2015;6:9924-36.

21. Zhou J, Wang C, Gong W, et al. uc.454 Inhibited Growth by Targeting Heat Shock Protein Family A Member 12B in Non-Small-Cell Lung Cancer. Mol Ther Nucleic Acids 2018;12:174-83.

22. Chen $W$, Liu X, Yuan S, et al. HSPA12B overexpression induces cisplatin resistance in non-small-cell lung cancer by regulating the $\mathrm{PI} 3 \mathrm{~K} / \mathrm{Akt} / \mathrm{NF}-\kappa \mathrm{B}$ signaling pathway. Oncol Lett 2018;15:3883-9.

23. Zoppino FCM, Guerrero-Gimenez ME, Castro GN, et al. Comprehensive transcriptomic analysis of heat shock proteins in the molecular subtypes of human breast cancer. BMC Cancer 2018;18:700.

24. Wang Y, Huang Y, Xu X, et al. Expression of small glutamine-rich TPR-containing protein A (SGTA) in 
Non-Hodgkin's Lymphomas promotes tumor proliferation and reverses cell adhesion-mediated drug resistance (CAM-DR). Leuk Res 2014;38:955-63.

25. Chao MP. Treatment challenges in the management of relapsed or refractory non-Hodgkin's lymphoma - novel and emerging therapies. Cancer Manag Res 2013;5:251-69.

26. Lwin T, Crespo LA, Wu A, et al. Lymphoma cell adhesion-induced expression of $\mathrm{B}$ cell-activating factor of the TNF family in bone marrow stromal cells protects non-Hodgkin's B lymphoma cells from apoptosis. Leukemia 2009;23:170-7.

27. Huang Y, Wang Y, Tang J, et al. CAM-DR: Mechanisms, Roles and Clinical Application in Tumors. Front Cell Dev Biol 2021;9:698047.

28. Huang $X$, Wang $Y, \operatorname{Nan} X$, et al. The role of the orphan G protein-coupled receptor 37 (GPR37) in multiple myeloma cells. Leuk Res 2014;38:225-35.

29. Wang X, Wang C, Qin YW, et al. The association of upregulation of $\mathrm{X}$-linked inhibitor of apoptosis protein with cell adhesion-mediated drug resistance in U937 cells. Hematol Oncol 2008;26:21-6.

30. Kurland JF, Kodym R, Story MD, et al. NF-kappaB1 (p50) homodimers contribute to transcription of the bcl-2 oncogene. J Biol Chem 2001;276:45380-6.

31. Ullah TR. The role of CXCR4 in multiple myeloma: Cells' journey from bone marrow to beyond. J Bone Oncol 2019;17:100253.

32. Kiang JG, Tsokos GC. Heat shock protein $70 \mathrm{kDa}$ : molecular biology, biochemistry, and physiology. Pharmacol Ther 1998;80:183-201.

33. Hu G, Tang J, Zhang B, et al. A novel endothelial-specific heat shock protein HspA12B is required in both zebrafish development and endothelial functions in vitro. J Cell Sci 2006;119:4117-26.

34. Cui Z, Wang P, Sun L, et al. Lipopolysaccharideevoked HSPA12B expression by activation of MAPK cascade in microglial cells of the spinal cord. J Neurol Sci 2010;294:29-37.

35. Hoffman AE, Demanelis K, Fu A, et al. Association of

Cite this article as: Huang Y, Peng C, Tang J, Wang S, Yang F, Wang Q, Zhou L, Yang L, Ju S. The expression of heat shock protein A12B (HSPA12B) in non-Hodgkin's lymphomas. Ann Transl Med 2021;9(18):1462. doi: 10.21037/atm-21-4185
AMP-activated protein kinase with risk and progression of non-Hodgkin lymphoma. Cancer Epidemiol Biomarkers Prev 2013;22:736-44.

36. Hosny G, Farahat N, Hainaut P. TP53 mutations in circulating free DNA from Egyptian patients with nonHodgkin's lymphoma. Cancer Lett 2009;275:234-9.

37. Paydas S, Ergin M, Erdogan S, et al. Prognostic significance of EBV-LMP1 and VEGF-A expressions in non-Hodgkin's lymphomas. Leuk Res 2008;32:1424-30.

38. Minoia C, Quero C, Asselti M, et al. Changes in angiogenesis and hypoxia-inducible factor- $1 \alpha$ protein expression in relapsed/refractory indolent non-Hodgkin lymphomas. Br J Haematol 2013;163:640-5.

39. Aggarwal D, Srivastava G, Gupta R, et al. Angiogenesis in Non-Hodgkin's Lymphoma: An Intercategory Comparison of Microvessel Density. ISRN Hematol 2012;2012:943089.

40. Elsamany S, Farooq MU, Elsirafy M, et al. Phase II study of low-dose fixed-rate infusion of gemcitabine combined with cisplatin and dexamethasone in resistant nonHodgkin lymphoma and correlation with Bcl-2 and MDR expression. Med Oncol 2014;31:872.

41. Ma Y, Lu C, Li C, et al. Overexpression of HSPA12B protects against cerebral ischemia/reperfusion injury via a PI3K/Akt-dependent mechanism. Biochim Biophys Acta 2013;1832:57-66.

42. Dehghanifard A, Kaviani S, Abroun S, et al. Various Signaling Pathways in Multiple Myeloma Cells and Effects of Treatment on These Pathways. Clin Lymphoma Myeloma Leuk 2018;18:311-20.

43. Hussain AR, Ahmed SO, Ahmed M, et al. Cross-talk between NFkB and the PI3-kinase/AKT pathway can be targeted in primary effusion lymphoma (PEL) cell lines for efficient apoptosis. PLoS One 2012;7:e39945.

44. Zhu MM, Wang L, Yang D, et al. Wedelolactone alleviates doxorubicin-induced inflammation and oxidative stress

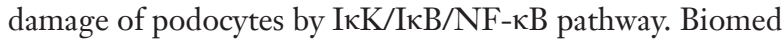
Pharmacother 2019;117:109088. 
Supplementary

Table S1 Summary of archival lymphoma samples tested using IHC, showing the percentage of samples positive for HSPA12B

\begin{tabular}{lcc}
\hline Tissue type & Total no.studied & IHC positive, $\mathrm{n}(\%)$ \\
\hline Normal lymph nodes & 23 & $22(95.65)$ \\
B cell lymphoma & 82 & $34(41.46)$ \\
DLBCL & 43 & $11(25.58)$ \\
FL & 23 & $18(78.26)$ \\
MALT & 16 & $5(31.25)$ \\
T cell lymphoma & 24 & $3(12.50)$ \\
NK/T & 24 & $3(12.50)$ \\
\hline IHC, immunohistochemistry; DLBCL, diffuse large B-cell \\
$\begin{array}{l}\text { lymphoma; FL, follicular lymphoma; MALT, extranodal lymphoma } \\
\text { of mucosa-associated lymphoid tissue; NK/T, nasal natural killer } \\
\text { (NK)/T-cell lymphoma. }\end{array}$
\end{tabular}

